\title{
JAZZIMPROVISATION ZWISCHEN SPONTANER ERFINDUNG UND ERLERNTEN MODELLEN. EIN EMPIRISCHER ANSATZ ZUR ANALYSE MIT HILFE VON VIDEO-STIMULATED-RECALL-INTERVIEWS
}

\section{Andreas Back und Peter Klose}

Die Praxis der Jazzimprovisation ist länger schon Gegenstand wissenschaftlicher Betrachtungen (vgl. für einen Überblick Knauer 2004b, Pfleiderer 2004 sowie den Beitrag von Martin Pfleiderer in diesem Band), vorwiegend allerdings ästhetischer, musiktheoretischer und -analytischer Art (vgl. z.B. Jost 1975, Knauer 1990, DeVeaux 1990 sowie Knauer 2004a). Eine wichtige Rolle spielt dabei von Anfang an das Ziel, Improvisieren mit Hilfe von Theorie systematisch lehr- und lernbar zu machen (vgl. etwa Bohländer 1947 als frühes Beispiel). Von zahlreichen Jazzmusiker*innen existieren außerdem Aussagen zu ihrem individuellen Zugang zur Improvisation, die in Interviews oder für Biographien gesammelt wurden. ${ }^{1}$ Der Saxphonist Dave Liebman versucht in den 1970er Jahren mit seiner Band Lookout Farm als einer der ersten, die Prozesse der Improvisation in der Gruppe über harmonisch-melodische Fragen hinaus als Fallstudie zu thematisieren (Liebman et al. 1977). Versuche eines systematischen Zugriffs auf die Praxis der Improvisation mit Hilfe der Methoden qualitativ-empirischer Forschung sind dagegen relativ jung. Als Meilenstein kann Paul F. Berliners umfangreiche Studie Thinking in Jazz gelten, die auf langjähriger Vorarbeit basiert (Berliner 1994: 3). Weiterhin gibt es psychologische und soziologische Studien (Pressing 1988, Behne 1992, Wilson/MacDonald 2016, Figueroa-Dreher 2016). Das Methodenrepertoire ist aber längst noch nicht ausgeschöpft.

Improvisation ist eine von Regelmäßigkeit und Dynamik geprägte soziale Praxis. Aus dem Zugriff auf geteilte Wissensbestände resultieren Regelmäßigkeiten, die durch das prinzipiell nicht prädeterminierte Handeln einzelner

1 Eine Zusammenstellung von Aussagen einzelner Musiker*innen etwa findet sich im viel zitierten Hear Me Talkin' To Ya von Nat Shapiro und Nat Hentoff (1966). 
Akteure in der Situation des Musizierens konterkariert werden. Erst im Zusammenspiel beider Facetten ergibt sich Improvisation als Phänomen, das also weder allein auf stilkritische Analysen, noch allein auf Untersuchungen individueller psychischer Prozesse zurückgeführt werden kann. Dem Blick auf die Situation des Improvisierens kommt eine Schlüsselrolle bei der Erforschung dieser Praxis zu; die in Musiktheorie geronnene Musiziertradition und ihre die Wahrnehmung strukturierende Rolle sowie der sich in den letztlich gespielten Klängen materialisierte Sinn, wie er sich der Musikanalyse im Nachhinein erschließt, dürfen dabei aber nicht außen vor gelassen werden.

Wir möchten daher in diesem Artikel Video-Stimulated-Recall-Interviews als Methode zur Erforschung von Improvisation mit Fokus auf die Situation des Improvisierens sowie einige der damit gewonnenen Ergebnisse aus einer 2016 durchgeführten Studie zur Diskussion stellen. ${ }^{2}$ Dabei geht es uns nicht um das Präsentieren vermeintlich letztgültiger Antworten; wir möchten eher in der Rückschau vorstellen, welche Anregungen und Perspektiven sich für die künftige Erforschung von Improvisation aus dem Projekt ergeben.

Nach einer Einordnung der Studie in den Forschungsstand zur (nicht nur) empirischen Improvisationsforschung und der Darstellung des methodischen Designs der Studie folgt der kontrastierende Vergleich zweier Detailbeispiele, an dem sich interessante Aspekte für zukünftige Studien und Optimierungsmöglichkeiten in Bezug auf die Methode aufzeigen lassen. Im Anschluss an einen Überblick über weitere Ergebnisse steht daher ein Ausblick auf Potentiale und Desiderate zukünftiger qualitativ-empirischer Erforschung von Improvisation.

\section{Forschungsstand: Ansätze zur (empirischen) Improvisationsforschung}

Unter den Erklärungsansätzen bzw. Modellen zur Improvisation gibt es zum einen solche, die a priori, also präskriptiv fungieren, weiterhin deskriptive, die auf Grundlage von Datenmaterial Improvisation rekonstruieren, sowie solche Ansätze, die versuchen, der Logik von Improvisation in actu nahezukommen. Diese Systematik soll im Folgenden anhand einzelner Beispiele erläutert werden, ohne dass der Anspruch eines erschöpfenden Überblicks in diesem Rahmen erfüllt werden kann.

2 Andreas Back (2016) hat diese Studie als Master-Arbeit im Rahmen seines Lehramtsstudiums an der TU Dortmund durchgeführt; Peter Klose war Betreuer und Erstgutachter. 
Zu den präskriptiven Entwürfen sind vor allem Improvisationsanleitungen im engeren Sinn zu zählen. Es handelt sich dabei in der Regel nicht um wissenschaftliche Modelle. Ein umfassender und gleichzeitig einflussreicher früher Entwurf ist z.B. George Russells Lydian Chromatic Concept of Tonal Organization (1959). Auf der Grundlage ausführlicher musiktheoretischer Überlegungen systematisiert Russell die Assoziation von Akkorden mit dazugehörigen Skalen. Diese Sichtweise auf das Improvisieren über Changes prägt bis heute das Erlernen und Unterrichten von Jazz als Idiom und Praxis. Ein anderes Beispiel sind Konzepte, die mit Hilfe z.B. pentatonischer Skalen eine Strategie zur Auswahl von Tönen beim Improvisieren bereitstellen. Beispiele hierfür sind etwa Werner Pöhlerts Basic Mediantic (1992) und die Pentatonic Khancepts des Gitarristen Steve Khan (2002).

Zwar sind solche Improvisationsanleitungen kaum ohne vorausgehende Erfahrungen in der Jazzpraxis denkbar und insofern in den seltensten Fällen rein präskriptiv - alle genannten Autoren sind in diesem Sinne auch Praktiker. Dennoch bilden musiktheoretische Überlegungen einen Einflussfaktor, der nicht aus den Spielerfahrungen allein resultiert. Als Improvisationsmodelle (und nicht bloß Modelle einer Jazzmusiktheorie) können sie darüber hinaus deswegen angesehen werden, weil sie eine Definition von Improvisation als primär musiktheoretisches Problem der Auswahl geeigneter Töne implizieren. Indem sie ein Teil eines Systems von »strukturierten und strukturierenden Dispositionen« (Bourdieu 2015: 97) werden, prägen sie aber »Wahrnehmungs-, Denk- und Handlungsschemata « (ebd.: 101) und werden so in der Situation des Improvisierens auf eine Weise wirksam, die über ihre Rolle bei der rationalen Entscheidung über die zu spielenden Töne hinausgeht. ${ }^{3}$

Zum anderen existieren Modelle, die wir als deskriptiv klassifizieren möchten. Das sind solche Ansätze, denen Datenmaterial aus bereits erfolgten Improvisationen zugrunde liegt - sei es als Transkription oder als Schallaufnahme. Hierzu zählen psychologische Erklärungsmodelle wie diejenigen von Jeff Pressing (1988) oder Klaus-Ernst Behne (1992). Weiterhin sind Herangehensweisen zu nennen, die durch musiktheoretisch fundierte Analysen Soli von Jazzmusikern auf Muster, Regelhaftigkeiten und idiosynkratische Elemente hin untersuchen. Martin Pfleiderers Jazzomat-Projekt hat zuletzt eindrucksvoll die Leistungsfähigkeit einer computergestützten Analyse eines großen Korpus im Rahmen solcher Untersuchungen bewiesen (Pfleiderer et al. 2017). Auf den algorithmischen Ansatz Philip N. Johnson-Lairds (1991, 2002)

3 Besonders bei instrumentenspezifischen Improvisationsanleitungen wirkt umgekehrt die Materialität des Musizierens auf das musiktheoretische Konstrukt zurück. 
möchten wir nicht inhaltlich, aber in Bezug auf die Herangehensweise etwas genauer eingehen.

Johnson-Lairds Methode ist synthetisch (im Gegensatz zu analytisch): Er rekonstruiert Improvisation als Prozess durch einen Algorithmus, d.h. eine prinzipiell programmierbare Verfahrensvorschrift. Die Validität dieses Algorithmus bemisst sich daran, ob er in der Lage ist, eine akzeptable Improvisation hervorzubringen, was in diesem Zusammenhang die Beurteilung anhand ästhetischer Maßstäbe bedeutet. Diese Vorgehensweise beruht daher zwangsläufig auf einem klar abgegrenzten Idiom; in diesem Fall ist dies die melodisch-harmonisch-rhythmische Konzeption des Bebop der 1940er Jahre, vorliegend in zahlreichen Transkriptionen und Aufnahmen. Dabei strebt Johnson-Laird eine Reichweite seines Modells bis auf die Ebene von Entscheidungen über konkrete Töne, Akkorde und Rhythmen an.

Johnson-Laird (1991: 319) schränkt aber schon ein, dass auch ein solcher im ästhetischen Sinn valider Algorithmus noch keine zwangsläufige Aussage über den Prozess des Improvisierens selbst erlaubt. Er schließt daher psychologische, letztlich kybernetische Überlegungen an, welche von mehreren denkbaren Formen von Algorithmen in Bezug auf den Menschen am plausibelsten erscheinen (ebd.; vgl. auch Johnson-Laird 2002: 242f.).

Bei Johnson-Laird zeigt sich das Potential deskriptiver Modelle, als Verfahrensvorschrift in präskriptive Anleitungen zum Improvisieren einzugehen. Man kann dies als systematisierte Erweiterung eines Lernens mit Hilfe von Transkriptionen ansehen, das im Jazz eine lange Tradition hat. Die Grundform dessen stellen transkribierte Soli wie Louis Armstrongs 50 Hot Chorusses for Cornet (1927) oder das Charlie Parker Omnibook (Parker 1978) dar; der nächste Schritt besteht im systematischen Ordnen (wie. z.B. in Form einer Sammlung von Jazzphrasen über die II-V-I-Verbindung, vgl. z.B. Kohl 2000), anschließend im Ableiten und Formulieren präskriptiver Regeln (vgl. Norgaard 2016). ${ }^{4}$

Weder präskriptive noch deskriptive Modelle können per se für sich in Anspruch nehmen, den Prozess des Improvisierens selbst valide zu beschreiben. Johnson-Lairds Ansatz wirft die Frage auf, ob die Gleichförmigkeit eines regelhaft ablaufenden Algorithmus den inneren Abläufen im Menschen bei einer Improvisation wirklich entspricht. Er hat sein Modell nicht umsonst an

4 Werke wie etwa Gunther Schullers Early Jazz (1986) bzw. The Swing Era (1991) oder Scott DeVeauxs The Birth of Bebop (2000) zählen wir dagegen nicht im engeren Sinne zu den deskriptiven Modellen, weil hier trotz zahlreicher Analysen improvisierter Soli der Fokus auf ästhetischen und stil- resp. bei DeVeaux sozialgeschichtlichen Entwicklung des Jazz im Sinne historisch-musikwissenschaftlicher Geschichtsschreibung liegt und nicht auf dem linear-prozesshaften Zustandekommen des einzelnen Solos. 
einem Programm getestet, das Walking-Bass-Linien erzeugt (Johnson-Laird 1991: 313ff., Johnson-Laird 2002: 437; dazu auch: Norgaard et al. 2013: 243f.). Diese Basslinien sind gleichförmig in ihrer rhythmischen Ausgestaltung. Sie sind aber insbesondere an die harmonische Grundlage gebunden. Auch wenn es zwar prinzipiell ein Spektrum vom bloßen Ausbuchstabieren der Akkorde bis hin zu geschmeidigen melodischen Linien mit Ausschmückungen und Durchgangstönen gibt, muss die Basslinie zumindest im Standard-Jazz trotzdem ein Mindestmaß an Regelmäßigkeit und Regelhaftigkeit aufbieten, das den Mitspielern den hörenden Nachvollzug der Changes erlaubt. JohnsonLaird liefert ein plausibles Modell für eine eng begrenzte Teilaufgabe im Rahmen des Improvisierens, wie sie das interne Prozessieren harmonisch-melodischer Regeln darstellt. Die darüber hinaus gehenden Abläufe in der sozialen Praxis des Improvisierens kann (und soll) es nicht abbilden.

Deskriptive Modelle betonen also tendenziell eher Regelhaftigkeit gegenüber der Dynamik und Offenheit für Neues bzw. Regelbrüche, die Improvisation prinzipiell innewohnt. Das heißt insbesondere, dass einige musikalische Idiome sich einer Modellierung durch einen Algorithmus möglicherweise stärker widersetzen.

Außerdem ergibt sich eine weitere erkenntnistheoretische und methodische Problematik in zweifacher Hinsicht: Erstens ist die wissenschaftliche Betrachtung von Improvisation anfällig für das, was Pierre Bourdieu den »scholastischen Irrtum« (Bourdieu 2017: 64) nennt: Das Modell, das sich Forschenden ohne Zeitdruck in Kontemplation und Reflexion darstellt, ist nicht mit der Logik der Praxis selbst zu verwechseln und stimmt vielleicht nur in Ausnahmefällen damit überein (Bourdieu 2015: 148f.). Für die sich in der Zeit entfaltende Praxis der Musik ist in diesem Zusammenhang z.B. bedeutsam, dass die synoptische Form der Transkription erlaubt, zeitlich nacheinander ablaufende Ereignisse »im selben Augenblick zu überblicken und damit ansonsten nicht wahrnehmbare Beziehungen (und unter anderem auch Widersprüche)« aufzudecken (ebd.: 151; vgl. dazu auch Jost 1975: 17f.). Zweitens muss man davon ausgehen, dass im Rahmen von Improvisation auch auf implizites, inkorporiertes Wissen zurückgegriffen wird, also auf die spraktischen, stummen, vorsprachlichen Könnens- und Erkennensformen sowie auf die im Zusammenspiel von Körpern beobachtbaren Koordinations-, Orientierungs- und Abstimmungsfähigkeiten« (Schmidt 2012: 59). Dabei ist zu fragen, in welchem Maße das inkorporierte knowing how des Improvisierens gänzlich auf die Automatisierung vormals bewussten Handelns zurückzuführen ist oder inwieweit es auch ein Residuum ebenso implizit erworbenen Körperwissens gibt, das beim Improvisieren zum Einsatz gebracht wird. Da Jazzimprovisation 
aber in der Regel an Standards und im Rückgriff auf die von Russell begründete Systematisierung mit Hilfe von Skalen gelehrt und gelernt wird, ist es plausibel, zumindest in diesem Bereich auch von einer Durchdringung von expliziten und impliziten Wissensbeständen auszugehen. ${ }^{5}$

Das führt zur dritten, logisch zwischen präskriptiven und deskriptiven Modellen angesiedelten Kategorie von Versuchen, der Improvisation in actu nahezukommen. Dazu genügt es nicht, auf Schallaufnahmen oder Transkriptionen zurückzugreifen; stattdessen muss man die Menschen in der Situation des Improvisierens selbst in den Blick nehmen - und das bedeutet methodisch: es müssen mindestens Videoaufnahmen benutzt werden. Besser noch sind teilnehmend-beobachtende, ethnographische Vorgehensweisen (denn auch eine Videoaufnahme ist nicht identisch mit der Situation selbst); zudem müssen - unter der Prämisse, dass wesentliche den Prozess leitende innere Vorgänge nicht beobachtbar sind - die beteiligten Musizierenden befragt werden. Sogar neurologische Methoden sind als Erweiterung denkbar.

Die Psychologen Graeme B. Wilson und Raymond A.R. MacDonald (2016) haben in diesem Zusammenhang eine qualitative Studie ähnlich der Backs durchgeführt. Sie haben Trios improvisierender Musiker*innen gefilmt, ihnen im Anschluss die Aufzeichnung gezeigt und dabei Interviews geführt. Die Probanden der Studie von Wilson und MacDonald sind so ausgewählt, dass sie eine künstlerische und stilistische Bandbreite repräsentieren, sollen aber ausdrücklich frei improvisieren. Dem liegt vermutlich die Hypothese zugrunde, dass freie Improvisation mehr Interaktion erfordert oder auch befördert (Wilson/MacDonald 2016: 1032). Der Fokus der Autoren liegt auf der Frage nach dem Zusammenhang von Interaktionen in der Gruppe und den individuellen Entscheidungs- bzw. Auswahlprozessen. Wilson und MacDonald entwerfen ein Modell des prozeduralen Flusses von Entscheidungen während des Improvisierens, das allerdings nicht die Ebene von Entscheidungen über einzelne Töne, Akkorde und Rhythmen berührt. Dem Modell liegt ein grundsätzlicher Zweischritt von Evaluation und anschließender Entscheidung über das als adäquat erachtete Handeln zugrunde. Die Ebenen der Entscheidung bestehen dann aus den Möglichkeiten zum Beibehalten oder Ändern des eigenen Improvisationsverhaltens, zum Reagieren oder Initiieren sowie auf musikalischer Ebene zur Übernahme, Erweiterung oder Kontrastierung des Gehörten (ebd.: 1035). Aus

5 Ein Modell, das explizit reflexives Handeln auf der Basis eines Handlungsentwurfs, automatisiertes, ehemals bewusstes Handeln sowie Handeln auf Basis von vorreflexiv-implizitem Wissen vereint, schlägt Gregor Bongaerts vor; er versucht damit, den Schütz'schen Handlungsbegriff mit praxeologischen Ansätzen zu verbinden (Bongaerts 2008: 227, 230 sowie Bongaerts 2007; vgl. dazu auch FigueroaDreher 2016: 103ff.). 
den Interviews mit den Probanden arbeiten Wilson und MacDonald anschließend die Kategorien bzw. Kriterien heraus, nach denen im Fluss des Improvisierens Entscheidungen getroffen werden (ebd.: 1036f.). Durch die Zweistufigkeit weist dieses Modell Ähnlichkeiten mit anderen ebenfalls psychologisch fundierten Improvisationsmodellen auf, die den Fluss der Improvisation als prinzipiell auflösbar in eine Abfolge von disjunkten Situationen verstehen, z.B. Pressing (1988) oder Behne (1992; vgl. dazu Figueroa-Dreher 2016: 47ff. sowie Pfleiderer 2004: 91f.).

Studien zum Prozess des Improvisierens finden sich - in Bezug auf die Frage nach Interaktion nicht völlig zufällig - auch auf Seiten der Soziologie. Ähnlich wie Wilson und MacDonald geht Silvana K. Figueroa-Dreher in ihrer sehr umfangreichen Studie vor. Ihre Probanden sind professionelle Free Jazzsowie Flamenco-Musiker (Figueroa-Dreher 2016: 143ff.); auch sie filmt die Musiker im Studio, interviewt sie im Rückgriff auf die Videoaufnahmen und entwickelt nach der Grounded Theory-Methodologie ein theoretisches Modell des Improvisierens (ebd.: 153ff.). Figueroa-Drehers Ansatz kann einer mundanphänomenologisch informierten Wissenssoziologie zugerechnet werden. Im Kontrast der beiden unterschiedlichen Musikstile arbeitet sie heraus, auf welche Weise die Musiker ihr Wissen zur Anwendung bringen. Interessant ist dabei der Unterschied der hochkomplexen Idiomatik des Flamenco, in der sich Regelhaftes und Freiheit verzahnen, und des insbesondere für musikalische Interaktion recht offenen Free Jazz (ebd.: 115, 264f.). Klänge spielen als musikalisches Material eine zentrale Rolle als Teil des Wissensbestands (ebd.: 173ff.), der Fokus liegt aber auf der Art und Weise des Zugriffs der Musiker auf dieses Material. Die Ebene der konkreten Entscheidungen etwa für bestimmte Töne wird auch von Figueroa-Dreher nicht berührt.

Der Soziologe und Saxophonist Peter Stegmaier (2012) wählt - ebenfalls mundanphänomenologisch informiert - einen anderen methodischen Zugriff: Er geht dem Prozess des Improvisierens lebensweltanalytisch-ethnographisch auf den Grund und setzt bei seinem eigenen Jazzspiel an. Anders als Wilson/MacDonald und Figueroa-Dreher widmet er sich dem Spiel von Jazzstandards, arbeitet aber auf einer übergeordneten Ebene das Charakteristische von Improvisation als »planvoll unplanvoll[es] Handeln« (ebd.: 339, 331f.) heraus. Kennzeichnend dafür sei, dass dem Unvorhergesehenen intentional ein Rahmen gesetzt wird - in verschiedenen Dimensionen: angefangen bei der Besetzung, der eigenen Rolle innerhalb der Band, der Stilistik, den Changes bis hin zu bewussten Handlungsentwürfen in Bezug auf das konkrete Solo (ebd.: 330).

Paul F. Berliners Thinking in Jazz (1994) stellt eine Zwischenstufe dar zwischen der deskriptiven Herangehensweise a posteriori und dem Versuch, 
die Logik der Praxis in actu zu erfassen. Er hat Musikerinnen und Musiker retrospektiv zum Prozess und zum Erlernen von Improvisation befragt. Das aus der Analyse der Interviews resultierende Modell konkretisiert er auf der musikalischen Ebene in Form von Analysen (ebd.: 11), tut dies aber im Rückgriff auf Aufnahmen. Gleichzeitig beruht die Studie auf umfangreicher ethnographischer Feldforschung in teilnehmender Beobachtung der Jazz-Szene. Der Unterricht, den Berliner bei verschiedenen Jazzmusikern nimmt, um seine eigenen Improvisationsfähigkeiten zu verbessern und entwickeln, spielt dabei eine doppelte Rolle: einerseits trägt er zum Verständnis der Erklärungen und Aussagen seiner Lehrer auch in (text-)analytischer Hinsicht bei, anderseits nutzt Berliner seine eigenen Improvisationserfahrungen als Sensibilisierung für Aspekte des Themas, ähnlich wie in Stegmaiers autoethnographischer Herangehensweise (ebd.: 10). Ein solcher Mixed Methods-Ansatz erscheint auch angesichts der Problematik einer klaren Definition von Improvisation sinnvoll. Berliner schreibt: „Was a particular practice improvisation or not? If one defines improvisation in such a way as to include the practice, then, presumably, it is « (ebd.: 4). Aus dieser Unmöglichkeit, deduktiv eine klare Definition von Improvisation zur Basis ihrer empirischen Erforschung zu machen, zieht Berliner für sich den Schluss, auch Praktiken des Jazz im größeren Rahmen in den Blick zu nehmen, um ihre möglicherweise subtilen Verbindungen zur Praxis der Improvisation nicht zu übersehen (ebd.: 9, 12).

Die im folgenden dargestellte Studie ist ebenfalls der dritten Kategorie zuzuordnen. Sie stellt einen Versuch dar, einerseits eng an der Situation des Improvisierens zu forschen und damit der Logik der Praxis nahezukommen, andererseits in Bezug auf die Musik auch den konkreten Entscheidungen über Klänge auf den Grund zu gehen.

\section{Fragestellung und Methodik des Projekts}

Jazzimprovisation bewegt sich im Spannungsfeld zwischen spontaner Erfindung und erlernten Modellen (Pfleiderer 2004, Johnson-Laird 2002, Knauer 2004), was diese Praxis einerseits dem Vorwurf aussetzt, dass Improvisationen aus vorgefertigten und nur scheinbar kreativen Elementen bestünden. Andererseits wird vielfach die spontane Kreation von Ideen hervorgehoben. Was genau an einer Jazzimprovisation ist aber nun eigentlich improvisiert? Diese etwas paradox anmutende Ausgangsfrage stand am Anfang der qualitativempirischen Studie von Andreas Back (2016). Mit Hilfe von Video-StimulatedRecall-Interview sollte im Anschluss an ein Konzert einer speziell ausgewählten Band durch die Rekonstruktion handlungsleitender Kognitionen der 
Unterschied zwischen dem Rückgriff auf Vorwissensbestände und spontaner Erfindung herausgearbeitet werden.

Die Studie geht von der Hypothese aus, dass das handlungsleitende Improvisationswissen der Probanden, das offengelegt werden soll, teilweise ein praktisches, inkorporiertes Wissen und teilweise explizites Wissen ist. Der Versuch des Zugangs zur Praxis im Moment des Handelns steht dabei im Vordergrund der Untersuchung.

Als Probanden haben sich Mitglieder der Band Filou aus Dortmund bereiterklärt. Die Band wurde im Jahr 2013 zunächst als Trio gegründet und hatte sich bis zum Zeitpunkt der Untersuchung zu einem größeren Ensemble bestehend aus einem Schlagzeuger, einem Bassisten, zwei Keyboardern, einem Gitarristen und einem Saxophonisten entwickelt. Bekannt geworden ist die Band durch einen Auftritt als Vorband der Band Snarky Puppy, die Konzertreihe Filou presents... im Dortmunder Club domicil sowie den Gewinn des futuresounds-Wettbewerbs und einen Auftritt im Hauptprogramm der Leverkusener Jazztage. Die interviewten Musiker haben ein Jazzstudium absolviert, verfügen über jahrelange Erfahrungen im Bereich der Jazzimprovisation und können als Experten auf diesem Gebiet angesehen werden. Der Stil der Band lässt sich zum Genre Fusion zählen, da er Elemente aus Jazz, Funk und HipHop enthält (vgl. Kirsten 2018). Es handelt sich also um eine Mischung aus einem idiomatischen Genre und struktureller Freiheit, da der Stil zum einen an ein harmonisches Grundgerüst gebunden ist und zum anderen ein relativ freies Improvisieren ermöglicht.

Der Fokus der Untersuchung liegt auf der musikalischen Momentaufnahme. Die leitende Fragestellung war daher: "Wie kommt ein Musiker in einem bestimmten Moment zu dem, was er spielt?« In dieser Formulierung wird zum einen der schon von Berliner (1994: 4) thematisierte, u.U. zirkelschlüssige Rückgriff auf den Begriff Improvisation vermieden. Zum anderen ist die Frage offen für Vorwissensbestände, die z.B. von musiktheoretischem Denken sowie geteiltem Wissen über stilistische Konventionen geprägt sind, aber auch für Einflussfaktoren, die situationsbezogen und materiell z.B. aus der Interaktion in der Gruppe oder aus dem auf das Instrument bezogene Körperwissen der Musizierenden resultieren. Die Fragestellung zielt also darauf $a b$, ausgehend von der Betrachtung der Schlüsselsituation des Improvisierens auch die präskriptive und die deskriptive Perspektive nicht von vornherein auszuschließen. 


\subsection{Methodik}

Beim Video-Stimulated-Recall-Interview schauen sich Probanden einen Ausschnitt eines Videos bezüglich ihres Verhaltens in einer bestimmten Situation an und reflektieren anschließend ihre Entscheidungsfindungsprozesse, die sie während der Aufnahme durchlaufen haben. Mit dieser Methode sollen Daten über Kognitionen von Individuen in einer bestimmten Situation erhoben werden. Die Methode ermöglicht im Idealfall das Zurückholen der Gedanken, die ein Individuum im Moment einer spezifischen Situation hatte (Nguyen et al. 2013). Zur Sicherstellung der im Interview entwickelten »Rekonstruktion von Gedanken, Überzeugungen, Normen und Entscheidungen« (Messmer 2015) werden Aufzeichnungen und Transkriptionen angefertigt (vgl. ebd.)

Die Interviews der vorliegenden Studie sind als Experteninterviews konzipiert worden, da sie der »Rekonstruktion komplexer Wissensbestände« (Meuser/Nagel 2010: 457) dienen. In diesem Fall geht es um die Rekonstruktion von Handlungswissen aus dem Bereich der Jazzimprovisation. Außerdem wird das Experteninterview eingesetzt, um »Erfahrungswissen und die Faustregeln, wie sie sich aus der alltäglichen Handlungsroutine« (ebd.) ergeben, zu gewinnen. Prinzipiell zielen Experteninterviews also sowohl auf explizite Wissensbestände wie auch auf die Offenlegung von implizitem knowing how ab.

Die Experteninterviews basieren auf einem Leitfaden, der eine flexible Durchführung gestattet. Daraus ergibt sich der Vorteil, dass im Interview auf die in Bezug auf die Fragestellung relevanten Bereiche eingegangen und »eine Vergleichbarkeit mit anderen Interviews, denen derselbe Leitfaden zugrunde lag « (ebd.) ermöglicht werden kann. Zudem zielen die Experteninterviews auf einen Zugang zu einem Wissen, über das zwar nicht nur Experten allein verfügen, »das aber doch nicht jedermann bzw. jederfrau in dem interessierenden Handlungsfeld zugänglich ist « (ebd.: 460f.).

Im direkten Anschluss an ein Konzert am 20. Januar 2016 fanden Einzelinterviews mit drei verschiedenen Instrumentalisten (Gitarrist, Saxophonist und Keyboarder) der Band Filou in den Räumlichkeiten des Dortmunder Jazzclubs domicil statt, um möglichst gute und detaillierte Erinnerungen der Musiker an ihr Gespieltes zu ermöglichen und die gegenseitige Einflussnahme zu vermeiden.

Die Interviews liefen nach dem folgenden Schema ab: Nach einer Einführung in die Methodik bekamen die Improvisatoren Konzertszenen mit ihrer solistischen Beteiligung auf einem Laptop vorgespielt und entschieden i.d.R. selbst, zu welchem Moment sie etwas sagen möchten. Die Interviewten durften eine eigene Szenenauswahl treffen und das Video stoppen, sobald ihnen 
etwas zu dem einfiel, was sie in einem bestimmten Moment musikalisch machten. Die Auswertung der Interviews fand in Anlehnung an die Qualitative Inhaltsanalyse und die induktive Kategorienbildung nach Philipp Mayring und Eva Brunner statt (vgl. Mayring/Brunner 2010: 323-333, Mayring 2010: 601613).

\section{Beispiele aus den Ergebnissen}

\subsection{Verschiedene Herangehensweisen an das Outside-Spielen}

Wir haben zwei Beispiele aus den Interviews herausgesucht, die unserer Ansicht nach interessante Anknüpfungspunkte bieten. In beiden Fällen geht es auf musikalischer Ebene um das Phänomen des sog. Outside-Spielens, zu dem sich die beiden befragten Musiker aber durchaus unterschiedlich äußern.

So äußerte sich der Gitarrist nach der folgenden Stelle:

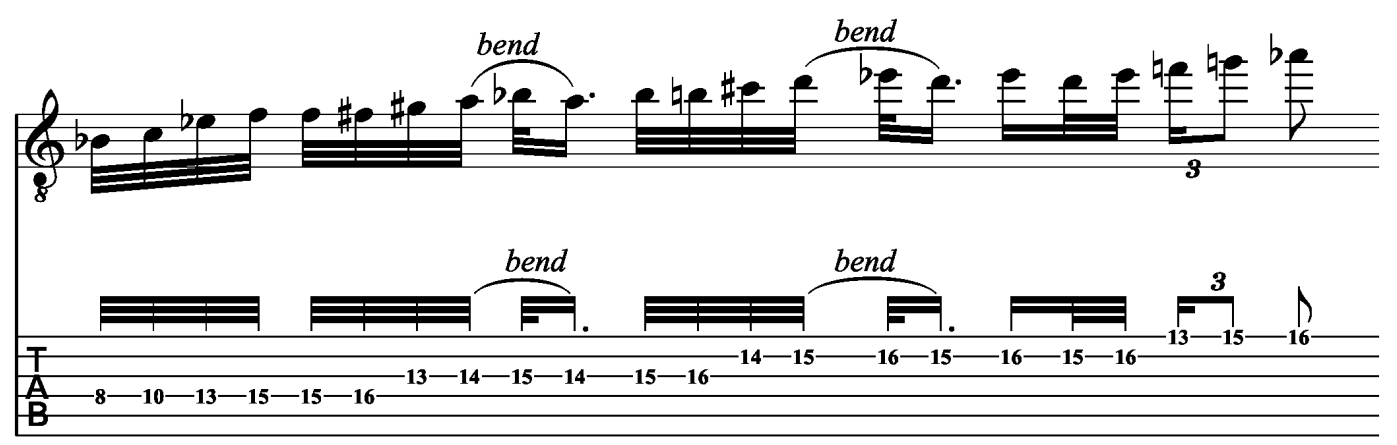

Nb. 1: Transkribierter Ausschnitt des Gitarrensolos

»[Gitarrist]: Chromatisch. Da bin ich quasi aus der Tonart rausgegangen. Da habe ich meine Homebase verlassen, kurz rausgegangen, dann wieder reingegangen. Und das sind Momente, wo man aber manchmal nicht weiß, wie das Zeug klingen wird. Wenn man jetzt einfach mal techt ${ }^{6}$ und einfach mal seine Finger machen lässt, aber bewusst, trotzdem bewusst rausgeht und weiß, wo man an welchen Tönen man plötzlich dann wieder rein muss.

[Interviewer]: Ok, das heißt, so ein großer Rahmen ist irgendwie, ist da, aber was dazwischen passiert ist dann, einfach so....

G.: ... ja (.) auch Risiko.

6 Als Verb abgeleitet von »Technik« bzw. vom englischen »technique«; gemeint: Instrumental- bzw. Spieltechnik in den Vordergrund der Improvisation stellen. 
I.: Risiko, okay

G.: Also die Kontrolle ist nicht mehr da« (Interview I, S. 2, Z. 12-21, Back 2016: 81).

Bedeutsam erscheint uns an der Stelle zum einen der Verweis auf das in Bezug auf die Musik planvoll-ungeplante Handeln, wie Stegmaier es nennt (»wo man manchmal nicht weiß, wie das Zeug klingen wird«). Gleichzeitig wird aber bewusst dem inkorporierten Wissen der Vorrang eingeräumt (»wenn man jetzt einfach mal techt und einfach mal seine Finger machen lässt«). Hier kommt die Gitarre als Instrument - oder in der soziologischen Terminologie: die Affordanz des Artefakts Gitarre - mit ins Spiel. Die zur Transkription in traditioneller Notation zusätzlich hinzugefügte Tabulatur soll daher die Fingersätze nachvollziehbar machen, die sich im Zusammenspiel von Körper und dem Griffbrett einer Gitarre im Standardtuning ergeben. Denn erst in der Interaktion mit der Gitarre vervollständigt sich das Handeln des Spielers. Methodologisch gesehen ist bei der Videographie also die Perspektive auf die Hände des Spielers von besonderer Bedeutung.

Eine ähnliche Stelle gibt es auch beim Saxophonisten, der aber in der Rückschau sein Tun ganz anders erklärt. Hier der Ausschnitt, auf den er sich bezieht:

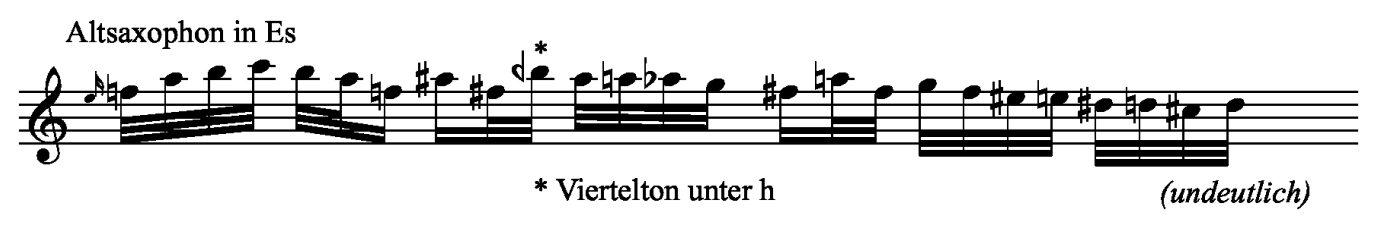

Nb. 2: Transkribierter Ausschnitt des Saxophonsolos

»Da haben wir so ein, so eine Outside-Konstruktion. Die habe ich schon mal häufiger von mir gehört irgendwie, weil das ist, wie gesagt, auch schnell, genau, und, ja, ist halt so ein Konzept, dass man... (2 [Sekunden Pause]) Ich fange jetzt hier an auf der Terz von dem Root quasi, also von dem D7, den ich da zu spielen habe. Da habe ich dann halt so eine Terz-Konstruktion da drüber gebaut. Das wäre dann Fis-Dur, der sich dann über F-Dur, quasi so halbtonmäßig runter, dann wieder auflöst, in das, was dann wieder passt. Genau « (Interview 3, S. 2, Z. 2-6, Back 2016: 93).

Es erscheint so, als seien in diesem Fall musiktheoretische Überlegungen leitend, die aufgrund des hohen Tempos dann aber mit Hilfe von Patterns umgesetzt werden. Bei der Interpretation des Interviewausschnitts ist nicht sicher zu entscheiden: Erläutert der Saxophonist seine eigenen präskriptiven Modelle, die ehemals bewusst angewendet wurden und durch Üben zu auto- 
matisierten Abläufen geworden sind (vgl. Bongaerts 2008: 227)? Oder analysiert er sein eigenes Spiel im Moment des Interviews a posteriori, was einer musikwissenschaftlichen Haltung gegenüber dem Spiel eines anderen Menschen gleichkäme? Oder hat er wirklich Zugriff auf die handlungsleitenden Kognitionen des Moments?

Geht man von einem Ineinandergreifen expliziten Wissens auf Grundlage bewusster Entscheidungen, automatisierten Routinen und habitualisiertem Handeln aus (vgl. ebd.: 229f.), dann schließen sich diese drei möglichen Interpretationen nicht zwangsläufig aus. Habitualisierter Umgang mit dem Instrument führt zu melodischen Erfindungen, denen anschließend reflexiv Sinn zugewiesen wird. Dieser Sinn ist dann die Grundlage weiterer bewusster Entscheidungen. Bei einem hohen Tempo wie im Beispiel beziehen sich solche Entscheidungen allerdings auf größere Einheiten und nicht auf einzelne Töne. Dies würde implizieren, dass die Aufmerksamkeit kontinuierlich zwischen der unmittelbaren Vergangenheit und der Vorausschau auf die unmittelbare Zukunft pendelt; Martin Norgaard (2011: 118) beschreibt eine solche Aufmerksamkeit auf das eigene Spiel als »evaluative monitoring «.

Interessant ist auch die Formulierung »die habe ich schon mal häufiger von mir gehört irgendwie «. Mit dieser Aussage wird bzgl. des eigenen Spielens die Perspektive eines Außenstehenden eingenommen. Es könnte sich hierbei einerseits um eine rhetorische Figur der (Selbst-)Darstellung im Rahmen der sozialen Situation Interview handeln. Andererseits bleibt genauer zu untersuchen, inwieweit die Einnahme eines solchen Beobachterstandpunkts gegenüber dem eigenen Tun sowohl auf der Mikro-Ebene im Moment des Improvisierens wie auch auf der Makro-Ebene der längerfristigen Entwicklung des eigenen Stils bedeutsam etwa im Sinne von Norgaards »evaluative monitoring « ist. Zu fragen wäre in diesem Zusammenhang auch, ob es dabei spezifische Strategien des Selbst-Befremdens gibt.

7 Methodologisch ergibt sich bei der hier geschilderten Vorgehensweise zusätzlich der Bedarf, den Zusammenhang zwischen musiktheoretischen Ausführungen der Probanden und der (Transkription der) Musik, auf die sich die Äußerungen beziehen, ggf. interpretierend zu rekonstruieren. 


\subsection{Kategorien von Improvisation zwischen planvollem und ungeplantem Handeln}

\section{Bewusste und unbewusste Entscheidungen}

Zunächst hat sich herausgestellt, dass alle drei Interviewten bewusste und unbewusste Entscheidungen im Improvisationsprozess auf direkte oder indirekte Art und Weise ansprechen. Bewusste Entscheidungen treffen die Musiker hauptsächlich über größere musikalische Strukturen. Dazu zählen alle Parameter, die eine Orientierung für das musikalische Vorgehen in einer Improvisation geben können wie etwa die Wahl der Klangfarbe, die Klangveränderung, die Nutzung von diversen Skalen und verschiedenem Tonmaterial, das Verlassen eines tonalen Zentrums, die Wahl bzw. das Bedienen eines konkreten Spielstils, der Aufbau eines Solos sowie melodische Entwicklungsprinzipien und Guide-Lines (vgl. Back 2016: 43f.).

Den Großteil der unbewussten Entscheidungen treffen die Improvisatoren über kleinere Einheiten innerhalb größerer musikalischer Strukturen einer Improvisation. Als Beispiel sei hier angeführt, dass die Musiker sich bewusst für die Wahl einer Klangfarbe oder die Nutzung von Skalenmaterial entscheiden, unbewusst hingegen für die Art und Weise der Klangerzeugung bzw. die konkrete Auswahl der Tonfolgen. Weitere unbewusste Prozesse der Improvisation beziehen sich auf das Entwerfen von Melodiefolgen beim Vertrauen auf die eigene Fingerfertigkeit, das Abrufen und die Auswahl von erarbeitetem Repertoire wie Licks und Ähnlichem (vgl. ebd.: 45-48).

\section{Verwendung von Bausteinen in einer Improvisation}

Alle drei Musiker äußern in den Interviews, dass sie bestimmte musikalische Bausteine in ihren Improvisationen nutzen, »die halt immer funktionieren und die [...] in das Raster dann eben so passen « (Interview 3, S. 3, Z. 14-15, Back 2016: 95). Mit Bausteinen sind also Elemente in einer Improvisation gemeint, die jederzeit abrufbar und in einem bestimmten Rahmen anwendbar sind. Dazu zählen Licks, Fills, Skalen, Ideenrepertoire u. Ä. Die Auswertung der Interviews legt den Schluss nahe, dass insbesondere in Stresssituationen oder für das Spielen schneller Läufe und Phrasen auf selbst erarbeitetes Improvisationsmaterial zurückgegriffen wird. Ein befragter Musiker vergleicht die musikalische Ausdrucksweise in einer Improvisation mit der sprachlichen Ausdrucksweise: Ist man rhetorisch gewandt, kann man sich gut ausdrücken. Um 
sich improvisatorisch adäquat ausdrücken zu können, werde ein ausreichendes Ideenrepertoire benötigt (ebd.: 48).

Bei der Betrachtung der Ergebnisse über die Verwendung von musikalischen Bausteinen in einer Improvisation fällt auf, dass die Musiker den Bausteinen unterschiedliche Funktionen zuweisen. Bausteine werden als Ausdrucksmittel, Rettungsanker in Stresssituationen, Füllmaterial zum Überbrücken von Ideenmangel, Lieferant für Ideenmaterial (Skalentöne) und virtuoses Element zur schnellen und technisch gewandten Ausführung eines Improvisationsabschnitts genannt (ebd.: 50).

\section{Einflussfaktoren auf eine Improvisation}

Von allen drei Teilnehmern der Untersuchung werden verschiedene Faktoren genannt, die sich in einer Improvisation auf ihr solistisches Spielen auswirken können. Im Rahmen der qualitativen Inhaltsanalyse konnten diese Faktoren den verschiedenen Bereichen formale Einflüsse, materielle Einflüsse, Einflüsse durch Mitspieler*innen und persönliche Einflüsse der Improvisierenden zugewiesen werden.

Formale Einflüsse beziehen sich im Wesentlichen auf die formale Anlage eines Stückes. Gewisse Tonarten und Harmoniefolgen grenzen beispielsweise die Auswahl des Ideenrepertoires ein oder erweitern sie, da sich die Spielerfahrungen und -gewohnheiten bezogen auf bestimmte Tonarten und Changes unterscheiden. Dies hat zur Folge, dass in manchen Improvisationen ein gröBerer und in manchen ein kleinerer Fundus an nutzbarem Improvisationsmaterial in Form von bereits verwendeten und passenden Motiven, Licks u. Ä. zur Verfügung steht (vgl. ebd.: 51).

Ferner wird durch das Arrangement vorgegeben, ob es offene oder zeitlich begrenzte Soli gibt, sodass im ersten Fall ein behutsamer Aufbau und eine behutsame Entwicklung eines Solos in Betracht kommen, während im zweiten Fall die Improvisationsfreiheit dadurch eingeschränkt wird, dass der Improvisierende unter dem Druck steht, innerhalb einer vorgegebenen Zeit sein improvisatorisches Können unter Beweis zu stellen. Ebenso begünstigen langsame Improvisationstempi die Kreativität bezüglich der Entwicklung von Phrasen, was durch die folgende Aussage eines Befragten unterstrichen wird: »Die schöneren Phrasen sind natürlich dann wieder da, wo man eigentlich Zeit hat, zu überlegen« (Interview 3, S. 6, Z.33-34, ebd.: 97).

Auch der Stil des Arrangements beeinflusst die Musiker in ihren Improvisationen, indem sie etwa die Wahl des Ton- und Skalenmaterials darauf ausrichten, Klischees zu bedienen oder zu durchbrechen (vgl. ebd.: 52f.). 
Die materiellen Einflüsse umfassen die Beschaffenheit des Instrumentes sowie die allgemeine Gestalt und Akustik des Aufführungsraumes. Die Interviewten berichten davon, dass die Wahl von Tonverbindungen teilweise von der Bauform und der Konstruktion des Instrumentes abhängig ist. Ausschlaggebend hierfür seien motorisch angenehme oder unangenehme Grifffolgen bzw. Tastenkombinationen (vgl. ebd.: 53f.).

Als Einflüsse durch Mitspieler*innen können jegliche Formen von Steigerungen wie die Erhöhung der Begleitlautstärke oder das Neueinsetzen weiterer begleitender Mitspieler*innen, das Spielen von bestimmten BegleitVoicings in Kombination mit einem bestimmten Basston und die gesamte Spielstilistik der Band genannt werden (vgl. ebd.: 54f.).

Des Weiteren können sich persönliche Faktoren auf eine Improvisation auswirken. Diese schließen Gewohnheiten, persönliche Klangvorstellungen (s. iv), individuelle Vorerfahrungen durch eine spezifische Ausbildung im Bereich der Improvisation, einen persönlichen Bezug zum Arrangement oder auch die Anzahl der bereits durchgeführten Improvisationen in einem Stück, Emotionen und die Beherrschung des Instrumentes ein (Interview 3, S. 6, Z. 33-34, vgl. ebd.: 55-57).

\section{Leitideen einer Improvisation: Klangvorstellungen, theoretische Zugänge}

Die Analyse der Interviews lässt den Schluss zu, dass eine Improvisation stark von den individuellen Klangvorstellungen der jeweiligen Improvisierenden geleitet wird. Verhältnismäßig oft nennen die Befragten die Begriffe Klangvorstellung, Klangideal, Klang und Sound, wenn es um die Beschreibung ihrer Improvisationen geht. Insgesamt unterstreichen die Musiker in den Interviews den hohen Stellenwert von Klangvorstellungen in einer Improvisation: Einerseits haben sie konkrete Assoziationen wie einen schreienden oder mixolydischen Klang als Klangvorstellung und andererseits verbinden sie Harmonien oder Skalen mit bestimmten Klangvorstellungen (vgl. ebd.: 57f.).

Als eine weitere Kategorie leitender Ideen haben sich theoretische $\mathrm{Zu}$ gänge zu einer Improvisation herausgestellt. Alle Improvisierenden nutzen im Vorhinein oder im Verlauf einer Improvisation theoretische Überlegungen, um eine Eingrenzung des zu gebrauchenden Tonmaterials vorzunehmen. Es fallen Aspekte wie Outside-Konstruktionen, Terz-Konstruktionen, Ausbrechen aus Schemata, Nutzung von Akkorderweiterungstönen und sogenannten upper structures sowie die passende Skalenwahl zu den Begleitharmonien (vgl. ebd.: 58-60). 


\section{Neuartiges in Form von spontanen Ideen}

In den Videomitschnitten und den Interviews finden sich Hinweise auf spontan entstandene Ideen, die sich in drei Klassen einteilen lassen: spontan erdachte Phrasen, allgemeine Inspirationen und spontane Ausführung von Erlerntem.

Mit spontan erdachten Phrasen werden musikalische Phrasen bezeichnet, die in einer Improvisation neu kreiert werden. Dies lässt sich möglicherweise in der Terminologie von Alfred Schütz als eine reflexive Ausdeutung spontanen Verhaltens (in Form eines Zusammenspiels von Körper und Musikinstrument) verstehen, das durch die umgehende Sinnzuweisung im Fluss des Spielens zum musikalischen Handeln wird. Eine aus implizitem, inkorporiertem knowing how des Instrumentalspiels resultierende Folge von Klängen wird auf diese Weise direkt im Anschluss durch Reflexion mit einem Handlungsentwurf in Einklang gebracht, der auch als um-zu-Motiv für weitere Musizierhandlungen trägt (vgl. Bongaerts 2008: 228f.).

Unter allgemeinen Inspirationen werden spontane Gedanken zur Gestaltung des Improvisierens aus zuvor gespielten Phrasen oder Motiven verstanden. Damit können Wiederholungen von Motiven, die Wahl des Tonmaterials und der Tonlage sowie die Umsetzung von Klangvorstellungen gemeint sein. Die spontane Ausführung von Erlerntem umfasst überwiegend die spontane Anwendung der oben beschriebenen musikalischen Bausteine einer Improvisation (vgl. Back 2016: 60f.).

\section{Fazit und Ausblick}

Auch wenn die vorliegende Studie schon allein vom Umfang her nicht als zuverlässige Validierung von Befunden und Hypothesen anderer Forschung herangezogen werden kann, bietet sie dennoch Hinweise und Anknüpfungspunkte für eine weitere qualitativ-empirische Erforschung von (Jazz-)Improvisation, und zwar sowohl in methodologischer wie inhaltlicher Hinsicht.

Es kann festgehalten werden, dass sich die Methode des Video-Stimulated-Recall-Interviews dazu bewährt hat, dem inkorporierten und implizierten Improvisationswissen ebenso wie den expliziten Wissensbeständen einzelner Musiker auf die Spur zu kommen und einen Zugang zur musikalischen Praxis des Improvisierens zu finden. Den Musikern scheint es mit Hilfe der Videoaufzeichnungen gelungen zu sein, ihr musikalisches Handeln und die den Improvisationsprozess leitenden Gedankengänge zumindest in Teilen zu rekonstruieren und zu reflektieren. Allerdings hat sich im Nachhinein gezeigt, dass 
nicht immer eindeutig zwischen der Rekonstruktion der handlungsleitenden Kognitionen und einer Reflexion des eigenen Handelns getrennt werden kann: Es ist nicht eindeutig zu beurteilen, ob der Interviewte gerade seine Gedankengänge während des Improvisationsprozesses selbst beschreibt oder ob er sein eigenes musikalisches Handeln eher a posteriori aus einer Beobachterperspektive analysiert und erläutert. Hierzu bedarf es einer Weiterentwicklung der Methode.

Stegmaier weist in Bezug auf seine eigene autoethnographische Vorgehensweise darauf hin, dass nicht nur »das subjektive Wissen anderer Menschen dem Forschenden nicht gänzlich und nicht direkt zugänglich ist « (Stegmaier 2012: 314), sondern dies auch für den Versuch gilt, dem eigenen Wissen in actu auf die Spur zu kommen (ebd.; vgl. auch Figueroa-Dreher 2016: 160ff.). Vor diesem Hintergrund weisen unterschiedliche Forschungsperspektiven auf das Improvisieren in jeweils verschiedener Hinsicht Desiderate auf: Figueroa-Drehers etwa hat als forschende Nicht-Musikerin einen Blick von außen (ebd.: 161), Stegmaier ist methodologisch geschult, muss aber als Saxophonist und native der Praxis Jazz mit Mitteln des Befremdens arbeiten (Stegmaier 2012: 314) und die von Berliner befragten Jazzmusiker sind ebenso wie die in dieser Studie teilnehmenden Musiker zwar Experten ihrer eigenen Praxis, bei denen aber ungeklärt bleibt, wie weit sie zu ihrem eigenen Wissen Zugang haben. Eine Alternative und Ergänzung zu den bisherigen Herangehensweisen könnte also z.B. eine methodologische Schulung von Jazzmusiker*innen als Expert*innen sein, um sie anzuleiten, ihrer eigenen Expertise introspektiv - ähnlich Liebman et al. (1977) - auf den Grund zu gehen.

Des Weiteren könnte eine Veränderung der Interviewsituation in Erwägung gezogen werden: Wenn den Befragten die Möglichkeit dazu eröffnet wird, ihre während der Aufzeichnung des Videos gespielten Phrasen auf dem eigenen Instrument nachzuspielen, könnte dies über die motorischen Abläufe möglicherweise die Rückerinnerung an die Improvisation unterstützen und ggf. optimieren. Hierbei könnte bei Instrumenten wie Gitarre oder Saxophon auch eine weitere Kameraperspektive hilfreich sein, die auf die Finger des jeweiligen Solisten ausgerichtet ist, ebenso wie eine aufwändigere Form der Audioaufnahme, die über getrennte Spuren auch im Nachhinein eine optimierte Tonqualität für das exakte Abhören jeden einzelnen Instruments erlaubt. Gegebenenfalls könnte weiterhin eine Kooperation mit Neurowissenschaftlern angestrebt werden, um qualitativ-empirisch gewonnene Ergebnisse z.B. mit Hilfe bildgebender Verfahren zur Untersuchung neuronaler Prozesse im Sinne einer Methodentriangulation zu untermauern.

In Bezug auf die Forschungsfrage bestätigt sich die Vermutung, dass Improvisationen größtenteils eine Kombination aus erlernten Modellen und 
spontanen Ideen sind. Die Befragten greifen explizit auf erlernte Modelle zurück, indem sie eine große Anzahl an bewussten Entscheidungen über die Stilistik, die Wahl des Tonmaterials in Form von Licks oder Skalen und das Ausbrechen aus einem tonalen Zentrum fällen. Solche theoretischen Vorwissensbestände erscheinen unabdingbar, wenn es um das Bedienen einer Spielstilistik, die Nutzung von zu einem Harmonieschema passenden Tonmaterial und um die bewusste Ausführung von Inside- und Outside-Spielen geht. Diese Rahmung der Improvisation durch teils auch schon a priori gefällte bewusste Entscheidungen fügt sich in Stegmaiers Prinzip: »planvoll unplanvoll Handeln« (ebd.: 339).

Erlernte Bausteine können im Rahmen einer Improvisation dagegen als vormals bewusstes, automatisiertes und insofern implizit verfügbares Wissen angesehen werden. Beispielsweise müssen gewisse Tonfolgen, Licks und Fills zunächst abgespeichert werden, damit sie in Improvisationen »als Ausdrucksmittel, Rettungsanker, Quelle für passendes Tonmaterial, Füllmaterial oder virtuoses Element einsetzbar sind.«(Back 2016: 63). Faktoren wie ein schnelles Improvisationstempo und die Vorerfahrungen mit einem bestimmten Stück begünstigen einen solchen Rückgriff auf Erlerntes. Es hat sich gezeigt, dass sich bei den Improvisierenden zum Teil unbewusst Gewohnheiten etablieren, indem in oft gespielten Stücken ähnliche Phrasen, Anfänge und Enden, GuideLines und ein ähnlicher Aufbau des Solos gewählt werden. In einem solchen Zusammenhang kann man also eher von spontaner Ausführung von bereits Erdachtem anstelle von unmittelbar spontaner Erfindung sprechen.

Andere Einflüsse können hingegen die spontane Erfindung bestärken. Während ein schnelles Improvisationstempo das Abrufen von erarbeitetem Repertoire begünstigt, fördert ein langsameres die spontane Kreation von Melodien und Phrasen. Darüber hinaus sind die Emotionen von Improvisierenden und die Einflüsse durch Mitspieler*innen, aus denen sich ein Wechselspiel zwischen Solist*in und Begleitband ergeben kann, der spontanen Erfindung zuträglich. Zudem werden die Solist*innen erfinderisch dadurch tätig, dass sie spontan einen Gesamtklang kreieren, der sich aus dem selbst erzeugten Klang und dem Klang der Begleitband zusammensetzt.

Ist das Ergebnis einer solchen Interaktion im Fluss des Spielens, bei der die Musiker*innen letztlich nicht wissen können, was die Mitspieler*innen im nächsten Moment spielen, reiner Zufall, oder lassen sich möglicherweise gerade hier auch Spuren implizit erworbenen und ebenso implizit angewendeten Körper- und Handlungswissens finden?

Für eine theoretische Grundlegung von Improvisationsforschung ist also weiterhin ein Begriff musikalisch-improvisatorischen Handelns nötig, der es erlaubt, das Wechselspiel zwischen implizitem, inkorporiertem knowing how 
und reflexivem Handeln auf der Basis von expliziten Wissensbeständen abzubilden. Gregor Bongaerts führt habituelles, präreflexives Wissen und den Handlungsbegriff von Schütz in einem Modell zusammen, das dafür geeignet erscheint (Bongaerts 2008: 230).

In Bezug auf den Unterschied zwischen einer musikalisch konventionalisierten Praxis von Jazz als Standard-Improvisation und dem in musikalischer Hinsicht geöffneten Free Jazz weist Stegmaier darauf hin, dass Free Jazz im handelnstheoretischen Sinn durchaus nicht frei ist (Stegmaier 2012: 338), so wie Ekkehard Jost dies schon 1975 für die strukturelle Dimension dieser Musik feststellt (Jost 1975: 11). Daran anknüpfend wäre es für ein zu entwerfendes musik- und improvisationsbezogenes Handlungsmodell also wichtig, zwischen den Dimensionen des musikbezogenen Handelns und der Dimension musikalischer Strukturen zu differenzieren. In beiden Dimensionen können sich in ganz unterschiedlichem Maße Regelmäßigkeiten und Konventionen sowie eine spezifische Dynamik entwickeln; nicht nur für die Musiker*innen, sondern auch in Bezug auf das Vorverständnis von Forscher*innen ist wichtig zu klären, in welcher Dimension man den Wesenskern dessen, was man als Improvisation ansieht, verortet. Die Auswahl der improvisierenden Probanden - in den vorliegenden Studien reicht das Spektrum von Flamenco über freie Musik und Free Jazz bis hin zu dem Spiel von Jazzstandards und Rock - ist daher auch methodologisch gesehen eine zentrale Entscheidung im Rahmen von Improvisationsstudien.

\section{Literatur}

Armstrong, Louis (1927). 50 Hot Choruses for Cornet. Chicago: Melrose Brothers.

Back, Andreas (2016). Jazzimprovisation zwischen spontaner Erfindung und erlernten Modellen. Eine empirische Studie. Unveröffentlichte Masterarbeit im Masterstudiengang für Lehramt an Gymnasien und Gesamtschulen an der Technischen Universität Dortmund.

Behne, Klaus-Ernst (1992). »Zur Psychologie der (freien) Improvisation. «In: Improvisation. 10 Beiträge. Hg. v. Walter Fähndrich. Winterthur: Amadeus, S. 42-62.

Berliner, Paul F. (1994). Thinking in Jazz. The Infinite Art of Improvisation. Chicago und London: The University of Chicago Press.

Bohländer, Carlo (1947). Harmonielehre für Jazz - Melodie - Improvisation. Frankfurt/M.: Selbstverlag.

Bongaerts, Gregor (2007). "Soziale Praxis und Verhalten - Überlegungen zum Practice Turn in Social Theory. «In: Zeitschrift für Soziologie 36 (4), S. 246-260.

Bongaerts, Gregor (2008). »Verhalten, Handeln, Handlung und soziale Praxis. «In: Phänomenologie und Soziologie. Theoretische Positionen, aktuelle Problemfelder und empirische Umsetzungen. Hg. v. Jürgen Raab, Michaela Pfadenhauer, Peter Stegmaier, Jochen Dreher u. Bernt Schnettler. Wiesbaden: Springer VS, S. 224232. 
Bourdieu, Pierre (2015 [1993]). Sozialer Sinn. Kritik der theoretischen Vernunft. Berlin: Suhrkamp.

Bourdieu, Pierre (2017 [2010]). Mediationen. Zur Kritik der scholastischen Vernunft. Berlin: Suhrkamp.

DeVeaux, Scott (2000). The Birth of Bebop. A Social and Musical History. London: Picador.

Figueroa-Dreher, Silvana (2016). Improvisieren. Material, Interaktion, Haltung und Musik aus soziologischer Perspektive. Wiesbaden: Springer VS.

Johnson-Laird, Philip N. (1991). »Jazz improvisation: A Theory at the Computational Level.«In: Representing Musical Structure. Hg. v. Peter Howell, Robert West und Ian Cross. London et al.: Academic Press, S. 291-326.

Johnson-Laird, Philip N. (2002). »How Jazz Musicians Improvise. «In: Music Perception 19 (3), S. 415-442.

Jost, Ekkehard (1975). Free Jazz. Stilkritische Untersuchungen zum Jazz der 60er Jahre. Mainz: Schott.

Khan, Steve (2002). Pentatonic Khancepts. Miami: Warner Brothers.

Kirsten, Sven (2018). »Filou«, http://www.filoumusic.de/, Zugriff: 5.4.2018.

Knauer, Wolfram (1990). Zwischen Bebop und Free Jazz. Komposition und Improvisation des Modern Jazz Quartet. 2 Bde. Mainz: Schott.

Knauer, Wolfram (Hg.) (2004a). Improvisieren... (= Darmstädter Beiträge zur Jazzforschung 8). Hofheim: Wolke.

Knauer, Wolfram (2004b). »Noodlin' and Doodlin' and Playin' Around... Zum sich wandelnden Selbstverständnis des Jazz als improvisierter Musik. « In: Improvisieren... Hg. v. dems. (= Darmstädter Beiträge zur Jazzforschung 8). Hofheim: Wolke, S. 19-38.

Kohl, Achim (2000). II-V-I Authentic Modern Jazz Phrasing. Schwetzingen: Schimper.

Liebman, Dave et al. (1977). Lookout Farm. A Case Study of Improvisation for Small Jazz Group. Alamo: Alamo Publications.

Mayring, Philipp (2010). »Qualitative Inhaltsanalyse. «In: Handbuch Qualitative Forschung in der Psychologie. Hg. v. Günter Mey und Katja Mruck. Wiesbaden: Verlag für Sozialwissenschaften, S. 601-613.

Mayring, Philipp / Brunner, Eva (2010). »Qualitative Inhaltsanalyse.«In: Handbuch Qualitative Forschungsmethoden in der Erziehungswissenschaft. Hg. v. Barbara Friebertshäuser, Antje Langer und Annedore Prengel. München u. Weinheim: Juventa, S. 323-333.

Messmer, Roland (2015). "Stimulated Recall als fokussierter Zugang zu Handlungsund Denkprozessen von Lehrpersonen. «In: Forum Qualitative Sozialforschung / Forum: Qualitative Social Research 16 (1), Art. 3, http://www.qualitativeresearch.net/index.php/fqs/rt/printerFriendly/2051/3732, Version vom 3.1. 2015, Zugriff: 5.4.2018.

Meuser, Michael / Nagel, Ulrike (2010). »Experteninterviews - wissenssoziologische Voraussetzungen und methodische Durchführung. « In: Handbuch Qualitative Forschungsmethoden in der Erziehungswissenschaft. Hg. v. Barbara Friebertshäuser, Antje Langer und Annedore Prengel. München u. Weinheim: Juventa, S. 457-471.

Nguyen, Nga Thanh / McFadden, Amanda / Tangen, Donna / Beutel, Denise (2013). »Video-stimulated Recall Interviews in Qualitative Research«, http://www. aare.edu.au/data/publications/2013/Nguyen13.pdf, Zugriff: 5.4.2018.

Norgaard, Martin (2011). »Description of Improvisational Thinking by Artistic-Level Jazz Musicians. «In: Journal of Research in Music Education 59 (2), S. 109-127.

Norgaard, Martin / Spencer, Jonathan / Montiel, Mariana (2013). »Testing Cognitive Theories by Creating a Pattern-Based Probabilistic Algorithm for Melody and 
Rhythm in Jazz Improvisation. « In: Psychomusicology: Music, Mind and Brain 23 (4), S. 243-254.

Norgaard, Martin (2016). »Unlocking Your Potential as an Improviser.«In: American String Teacher 66 (2), S. 26-29.

Parker, Charlie (1978). Charlie Parker Omnibook. Hg. von Michael H. Goldsen. New York: Atlantic Music.

Pfleiderer, Martin (2004). »Improvisieren - ästhetische Mythen und psychologische Einsichten. «In: Improvisieren.... Hg. von Wolfram Knauer (= Darmstädter Beiträge zur Jazzforschung 8). Hofheim: Wolke, S. 81-99.

Pfleiderer, Martin et al. (Hg.) (2017). Inside the Jazzomat. New Perspectives for Jazz Research. Mainz: Schott.

Pressing, Jeff (1988). »Improvisation: Method and Models. «In: Generative Processes in Music. The Psychology of Performance, Improvisation and Composition. Hg. v. John A. Sloboda. Oxford u. New York: Clarendon Press u. Oxford University Press, S. 129-178.

Pöhlert, Werner (1992). Basic Mediantic. Schwetzingen: Schimper.

Russell, George (1959). The Lydian Chromatic Concept of Tonal Organization for Improvisation: for all Instruments. Cambridge: Concept Publishing.

Schmidt, Robert (2012). Soziologie der Praktiken. Konzeptionelle Studien und empirische Analysen. Berlin: Suhrkamp.

Schuller, Gunther (1986 [1968]). Early Jazz. Its Roots and Musical Development. New York u. Oxford: Oxford University Press.

Schuller, Gunther (1990). The Swing Era. The Development of Jazz 1930-1945. New York u. Oxford: Oxford University Press.

Shapiro, Nat / Hentoff, Nat (Hg.) (1966). Hear Me Talkin' To Ya. The Story Of Jazz As Told By The Men Who Made It. New York: Dover.

Stegmaier, Peter (2012). »Phänomenologische und handelnstheoretische Reflektionen zum eigenen Jazzspiel als soziale Praxis. «In: Angewandte Phänomenologie. Zum Spannungsverhältnis von Konstruktion und Konstitution. Hg. v. Jochen Dreher. Wiesbaden: Springer, S. 311-342.

Wilson, Graeme B. / MacDonald, Raymond A. R. (2016). »Musical Choices During Group Free Improvisation: A Qualitative Psychological Investigation.« In: Psychology of Music 44 (5), S. 1029-1043.

\begin{abstract}
This article proposes video stimulated recall interviews as a method in research on improvisation. Assuming a categorization of approaches in prescriptive, descriptive and practice orientated models of improvisation the authors argue towards a focus on the actual situation of musical action and interaction without neglecting the formative role of shared knowledge concerning music theory, stylistic conventions and material conditions of improvisation. The article discusses video stimulated recall interviews on a methodological background in comparison to other studies. Furthermore, it presents findings of a recent qualitative empirical study by Andreas Back (2016): A fusion band was filmed on performance in a jazz club; three members were shown the recordings and interviewed right after the concert.
\end{abstract}

